\title{
Convention additionnelle à la convention tarifaire TARMED FMH-AA/AM/AI du 28 décembre 2001 concernant le tarif dédié aux médecins agréés*
}

entre les assureurs selon la loi fédérale sur l'assurance accident, représentés par La Commission des tarifs médicaux LAA (CTM), I'Office fédéral de l'assurance militaire (OFAM), I'assurance invalidité, représentée par l'Office fédéral des assurances sociales appelés ci-dessous assureurs, et la Fédération des médecins suisses (FMH), I'Association suisse des médecins indépendants travaillant en cliniques privées et hôpitaux (ASMI)

\section{Préambule}

Cette convention ne règle que les points divergeant de la convention tarifaire TARMED du 28 décembre 2001.

\section{Eléments de la convention}

Font partie intégrante de la convention les annexes suivantes: Annexe I (Déclaration personnelle/Critères de validité), Annexe II (Positions tarifaires additionnelles) et Annexe III (Facturation).

\section{Champ d'application}

La présente convention est applicable par les médecins agréés gérant leur propre cabinet selon le droit privé. Elle règle la compensation des frais généraux de cabinet dans le domaine AA/AM/AI lors de prestations interventionnelles fournies par les médecins agréés dans les établissements hospitaliers avec lesquels existe un accord de facturation à l'acte.

\section{Adhésion à la convention}

1 Chaque médecin agréé, répondant aux critères définis au point 3 , membre de l'ASMI et ayant adhéré à la convention tarifaire TARMED, peut adhérer à la présente convention en adressant sa déclaration personnelle (Annexe I) à l'ASMI.

* Vous trouverez ces documents aussi sous le lieu suivant: www. fmh.ch/ww/fr/pub/prestations/ tarifs/andere_tarife.htm
2 Les médecins agréés, non-membres de l'ASMI mais ayant adhéré à la convention tarifaire TARMED, peuvent adhérer à la présente convention. Ils adresseront leur demande accompagnée de la déclaration personnelle (Annexe I) au Service central des tarifs médicaux LAA (CTM) à Lucerne.

3 Les médecins agréés, non-membres de l'ASMI, devront s'acquitter d'une finance d'adhésion unique de Fr. 900.- (participation aux frais). L'adhésion à la convention devient effective lors du paiement de cette finance.

4 Le médecin agréé autorise la FMH de donner la possibilité aux assureurs de consulter ses données personnelles de la valeur intrinsèque.

\section{Compensation des frais généraux de cabinet (CFC)}

1 Le médecin agréé a droit, dans le cadre de cette convention, à une compensation des frais généraux de son cabinet (CFC) de 2.29 points/minute. Le minutage est déterminé par la position TARMED correspondante comprenant la prestation au sens restreint, la préparation, la finition et le rapport.

2 Dans le cadre de cette convention, l'assistant, lorsque le TARMED en prévoit un et qu'il s'agit d'un médecin agréé ayant adhéré à cette convention, a droit à une compensation de ses frais généraux (CFC) de 2.29 points/ minute; le minutage est celui de la position TARMED correspondante comprenant la prestation au sens restreint, la préparation, la finition et le rapport.

3 La compensation des frais de cabinet (CFC) est exprimée comme une position tarifaire additionnelle (Annexe II).

4 La valeur du point s'établit selon la convention concernant la valeur du point tarifaire du 28 décembre 2001 (partie intégrante de la convention tarifaire TARMED art. 2 al. 1 l. b.) 
5 Les médecins anesthésistes ne peuvent se prévaloir de cette compensation des frais de cabinet (CFC) car selon les chapitres 28 et $29 \mathrm{du}$ TARMED, leur prestation technique y est déjà réglée (cf. Annexe III)

\section{Stabilisation des coûts par cas}

1 En analogie à la convention du TARMED du 28 décembre 2001 concernant la stabilité des coûts par cas (partie intégrante de la convention tarifaire TARMED conformément à l'art 2 al 11 c), les coûts par cas sont observés pendant une durée maximale de 18 mois après l'entrée en vigueur de cette convention, respectivement jusqu'à l'introduction du tarif dédié aux médecins agréés. Dans le cadre d'une commission de contrôle propre, les médecins agréés feront l'objet d'une analyse en tant que groupe indépendant de médecins.

2 En cas d'atteinte à la stabilité des coûts par cas, les mécanismes de correction suivants pourront être mis en œuvre:

- correction de l'application de la position tarifaire 00.0210;

- correction du taux de la CFC;

- établissement de forfaits pour certaines positions de prestations.

\section{Facturation}

Le médecin agréé ayant adhéré à la convention établira ses notes d'honoraires selon l'Annexe III.

\section{Tarif dédié aux médecins agréés}

1 Les parties contractantes s'engagent à mettre sur pied, avant le 31 décembre 2005, un tarif applicable par les médecins agréés, reposant sur la structure TARMED, sous la forme de forfaits basés sur les indications les plus courantes dans le domaine AA/AM/AI. L'introduction de ce tarif peut se faire progressivement.

2 Les parties contractantes mettent au point, avant le 30 septembre 2004, le projet de l'élaboration du tarif dédié aux médecins agréés comportant au moins en partie les domaines suivants:
- une définition commune des interventions les plus fréquentes dans le domaine AA/AM/AI qui seront en priorité indemnisées sous la forme de forfaits liés à l'indication ou au diagnostic;

- la marche à suivre pour l'élaboration de ces forfaits;

- la définition commune des critères de qualité;

- la définition commune des critères d'efficacité, d'adéquation et d'économicité.

\section{Entrée en vigueur et durée de la convention}

1 Cette convention entre en vigueur avec effet rétroactif au $1^{\mathrm{er}}$ avril 2004.

2 Les honoraires conformément à cette convention ne pourront être établis qu'après le $1^{\text {er }}$ juillet 2004.

3 Cette convention peut être dénoncée pour la fin de chaque semestre moyennant un délai de six mois. La convention ne pourra pas être dénoncée avant le 31 décembre 2005.

\section{Annexes}

- Annexe I: Déclaration personnelle /Critères de validité;

- Annexe II: Positions tarifaires additionnelles (CFC);

- Annexe III: Facturation.

Berne/Lucerne, le 24 juin 2004

Fédération des médecins suisses (FMH) Le Président: H. H. Brunner

La Secrétaire générale: A. Müller Imboden

Association suisse des médecins indépendants travaillant en cliniques privées et hôpitaux ASMI

Le Président: B. Burri

Le Secrétaire: U. Wanner

Commission des tarifs médicaux (CTM) Le Président: W. Morger

Office fédéral de l'assurance militaire Le Directeur a.i.: K. Stampfli

Office fédéral des assurances sociales Assurance invalidité

La Vice-directrice: B. Breitenmoser 


\section{Annexe I}

\section{Déclaration personnelle}

Nom:

Prénom:

Adresse cabinet:

Téléphone:

EAN:

Titre de spécialiste FMH:

Activité en tant que médecin agréé dans les établissements hospitaliers suivants:

Membre de la FMH: non / oui

Adhésion à la convention tarifaire FMH-CTM/AA/AM/AI: non / oui, le

Membre de l'ASMI: non / oui

Si oui, la déclaration personnelle doit être adressée à l'ASMI.

Si non, la déclaration personnelle doit être envoyée dans l'enveloppe réponse ci-jointe au Service central des tarifs médicaux (CTM). La finance d'adhésion unique de Fr. 900.- doit être versée à la CMT par le médecin agréé non membre de I'ASMI pour adhérer à la convention additionnelle.

Valeur intrinsèque transmise à la FMH: non / oui

Le soussigné autorise la FMH de donner la possibilité aux assureurs fédéraux de consulter ses données personnelles de la valeur intrinsèque.

Attestation de la caisse AVS d'une activité professionnelle principalement indépendante: oui(non Le médecin agréé soussigné a jusqu'à présent facturé de manière indépendante ses prestations fournies en milieu hospitalier dans le domaine l'AA/AM/AI: oui / non

Les prestations hospitalières du médecin agréé soussigné dans le domaine AA/AM/AI ont été jusqu'à présent facturées par l'hôpital en même temps que les prestations de l’hôpital: oui / non

L'opérateur soussigné s'engage à transmettre les positions tarifaires utilisées lors de son activité hospitalière à l'anesthésiste et à l'hôpital.

Le soussigné s'engage à traiter les patients AA/AM/AI en fonction des capacités hospitalières et selon l'indication médicale: oui / non

Le soussigné confirme l'exactitude des indications et adhère à la convention additionnelle à la convention tarifaire TARMED FMH-CTM/AA/AM/AI du 28 décembre 2001 concernant le tarif dédié aux médecins agréés.

Lieu / Date:

Signature:

\section{Remarques importantes}

Les conditions à l'adhésion de la convention additionnelle pour le tarif des médecins agréés sont les suivantes:

- adhésion à la convention tarifaire TARMED entre la FMH et CTM/AA/AM/AI;

- affiliation à l'ASMI ou paiement d'une finance d'adhésion de Fr. 900.- à la CTM;

- enregistrement de la valeur intrinsèque dont les données sont consultables par les assureurs;

- traitement des patients AA/AM/AI en fonction des capacités hospitalières et selon l'indication médicale;

- attestation de la caisse AVS d'une activité professionnelle principalement indépendante. 
Annexe II

50.0010

Indemnisation des frais de cabinet (IFC) du médecin agréé pendant le traitement ambulatoire ou stationnaire de patients AA/AM/Al dans un hôpital employant des médecins agréés, par min.

\begin{tabular}{|c|c|c|c|c|c|}
\hline $\begin{array}{l}\text { Valeur } \\
\text { intrinsèque }\end{array}$ & $\begin{array}{l}\text { Valeur } \\
\text { intrinsèque } \\
\text { qualitative }\end{array}$ & $\begin{array}{l}\text { Unité } \\
\text { fonctionnelle }\end{array}$ & $\begin{array}{l}\text { Classe } \\
\text { de risque }\end{array}$ & & \\
\hline - & - & - & - & & \\
\hline $\begin{array}{l}\text { PM (assistance } \\
\text { comprise) } \\
\text { - }\end{array}$ & Assistance & $\begin{array}{l}\text { Nombre } \\
\text { d'assistants }\end{array}$ & $\begin{array}{l}\text { Prestation } \\
\text { au sens } \\
\text { restreint }\end{array}$ & $\begin{array}{l}\text { Préparation } \\
\text { et finition }\end{array}$ & Rapport \\
\hline PT & $\begin{array}{l}\text { Temps } \\
\text { d'occupation }\end{array}$ & $\begin{array}{l}\text { Temps } \\
\text { d'attente }\end{array}$ & & & \\
\hline $2,29 \mathrm{pts}$ & $1 \mathrm{~min}$ & - & & & \\
\hline
\end{tabular}

\section{Interprétation médicale}

Est répute temps imputable le temps fixé dans le tarif pour la prestation au sens restreint, la préparation et finition et le rapport.

N'est valable que pour les traitements opératoires/interventionnels ambulatoires ou stationnaires effectues à l'hôpital par le médecin agréé, ne recevant pas de rémunération fixe de l'hôpital, qui a adhéré à la convention entre la CTM/AM/AI et la FMH/Médecins agréés.

\section{Interprétation technique (règles)}

- 00.0210 ne peut être facturée qu'une fois par cas avec 50.0010.

- 00.0310 peut être facturée quatre fois au maximum avec 50.0010 le premier jour d'hospitalisation et deux fois par jour au maximum les jours d'hospitalisation suivants jusqu'au jour de sortie.

- Non applicable avec les prestations des chapitres $02,28,29,30,31,32$.
50.0020

\section{Indemnisation des frais de cabinet (IFC) pour l'assistance par le médecin agréé pendant le traitement ambulatoire ou stationnaire de patients AA/AM/AI dans un hôpital employant des médecins agréés, par min.}

\begin{tabular}{|c|c|c|c|c|c|}
\hline $\begin{array}{l}\text { Valeur } \\
\text { intrinsèque }\end{array}$ & $\begin{array}{l}\text { Valeur } \\
\text { intrinsèque } \\
\text { qualitative }\end{array}$ & $\begin{array}{l}\text { Unité } \\
\text { fonctionnelle }\end{array}$ & $\begin{array}{l}\text { Classe } \\
\text { de risque }\end{array}$ & & \\
\hline - & - & - & - & & \\
\hline $\begin{array}{l}\text { PM (assistance } \\
\text { comprise) } \\
\text { - }\end{array}$ & Assistance & $\begin{array}{l}\text { Nombre } \\
\text { d'assistants }\end{array}$ & $\begin{array}{l}\text { Prestation } \\
\text { au sens } \\
\text { restreint }\end{array}$ & $\begin{array}{l}\text { Préparation } \\
\text { et finition }\end{array}$ & Rapport \\
\hline PT & $\begin{array}{l}\text { Temps } \\
\text { d'occupation }\end{array}$ & $\begin{array}{l}\text { Temps } \\
\text { d'attente }\end{array}$ & & & \\
\hline 2,29 pts & $1 \mathrm{~min}$ & - & & & \\
\hline
\end{tabular}

\section{Interprétation médicale}

- Est réputé temps imputable le temps fixé dans le tarif pour la prestation au sens restreint, la préparation et finition et le rapport.

- N'est valable que pour les traitements opératoires/interventionnels ambulatoires ou stationnaires effectues a l'hôpital dans le cadre de l'assistance par le médecin agréé, ne recevant pas de rémunération fixe de l'hôpital, qui a adhéré à la convention entre la CTM/AM/AI et la FMH/Médecins agréés. Uniquement facturable dans la mesure où une assistance est prévue dans le tarif et où l'assistance par le médecin agréé a effectivement eu lieu pendant le traitement.

- Uniquement facturable pour la première assistance.

\section{Interprétation technique (règles)}

Non applicable avec les prestations des chapitres $02,28,29,30,31,32$.

\section{Annexe III}

\section{Facturation}

\section{Principe}

La facturation s'effectue suivant les modalités du contrat-cadre TARMED. En plus, le numéro EAN du médecin agréé assurant l'assistance doit également figurer sur la note d'honoraires. Les prestations du médecin agréé et celles de l'hôpital doivent être facturées séparément de même que les prestations ambulatoires au cabinet et les prestations ambulatoires et stationnaires à l'hôpital.

\section{Facturation}

Le médecin agréé qui a adhéré à la convention et remplit les conditions conformément au chiffre 3 de la convention, établit personnellement ou par l'intermédiaire d'un mandataire, une note d'honoraires pour les prestations ambulatoires en hôpital ou en cas d'hospitalisation de la manière suivante:

\section{Médecin agréé}

- Prestation médicale y compris assistance;

- compensation des frais généraux de cabinet (CFC), également pour l'assistant s'il s'agit d'un médecin agréé.

\section{Anesthésiste}

- Prestations médicale et technique pour le management péri-opératoire;

- prestation médicale lors de la préparation et la finition; 
- prestation médicale pour la durée de l'anesthésie.

La rémunération de l'assistant suivant cette convention incombe au médecin agréé responsable.

\section{Remboursement des honoraires}

Le remboursement des notes d'honoraires de l'opérateur et de l'anesthésiste, établies séparément mais en relation évidente, n'aura lieu que lorsque les deux seront parvenues aux assureurs concernés. C'est pour cette raison que les médecins agréés s'engagent à établir leur note d'honoraires au plus tard 30 jours après la fin du traitement à l’hôpital. 